\title{
Medical Expulsive Therapy for Pediatric Urolithiasis: Systematic Review and Meta-Analysis
}

\author{
Nermarie Velázquez, BS ${ }^{\star}$, Daniel Zapata, MD*, Hsin-Hsiao S. Wang, MD, MPH, John S. \\ Wiener, MD, Michael E. Lipkin, MD, and Jonathan C. Routh, MD, MPH \\ Division of Urologic Surgery, Duke University Medical Center, Durham, NC
}

\section{Summary}

Objective-Kidney stone disease has become more common among children and young adults. Despite its well-documented success in adults, published success rates of medical expulsive therapy (MET) for pediatric urolithiasis vary widely. Our objective was to determine whether the aggregated evidence supports the use of MET in children.

Methods-We searched the Cochrane Controlled Trials Register, clinicaltrials.gov, MEDLINE, and EMBASE databases, and recently presented meeting abstracts for reports in any language. In addition, the bibliographies of included studies were then hand-searched. The protocol was prospectively registered at PROSPERO (CRD42013005960). Inclusion criteria were children (aged $\leq 18$ years) with urolithiasis treated with medications with the specific goal of increasing spontaneous stone passage rate, including but not limited to alpha-adrenergic blockers (e.g., tamsulosin or doxazosin), calcium channel blockers (e.g., nifedipine), or other adjuvant medications (e.g., steroids or tolterodine). Manuscripts were then assessed and data abstracted in duplicate, with differences resolved by the senior author. Risk of bias was assessed using standardized instruments. Descriptive statistical analyses were performed as appropriate.

Results-We identified 11,197 studies, five of which (3 randomized controlled trials, 2 retrospective cohorts) were included in the pooled meta-analysis. Although we found little evidence of significant publication bias, we were unable to assess the likelihood of other forms of bias (allocation, selection) for most included studies due to reporting limitations. The pooled results demonstrate that MET significantly increased the odds of spontaneous stone passage (OR $2.21,95 \%$ CI $1.40-3.49)$. Between-study heterogeneity was not statistically significant $\left(I^{2}=14 \%\right.$, $p=0.36$ ). Bivariate meta-regression models revealed no significant association between the likelihood of stone passage and study COI $(p=0.9)$, study country $(p=0.7)$, patient age $(p=0.4)$, patient gender $(p=0.4)$, duration of follow-up $(p=0.3)$, or stone size $(p=0.7)$. Side effects of MET were reported to be minimal. Relatively few patients reported any adverse effects at all; the

Corresponding Author: Jonathan C. Routh, MD, MPH, Division of Urologic Surgery, Duke University Medical Center, DUMC 3831, Durham, NC 27710, TEL: 919-684-6994, FAX: 919-681-5507, Jonathan.Routh@ duke.edu.

Both authors contributed equally to this paper.

Publisher's Disclaimer: This is a PDF file of an unedited manuscript that has been accepted for publication. As a service to our customers we are providing this early version of the manuscript. The manuscript will undergo copyediting, typesetting, and review of the resulting proof before it is published in its final citable form. Please note that during the production process errors may be discovered which could affect the content, and all legal disclaimers that apply to the journal pertain.

Financial Disclosure:

Conflict of Interest: The remaining coauthors have no financial relationships relevant to the article to disclose. 
most commonly reported issue was somnolence. Concerns about biases affecting the published outcomes of the included studies exist due to the low quality of the randomized controlled trials reviewed for analysis. However, there was little visual evidence of publication bias noted on the funnel plot, as confirmed by the Begg test $(p=0.5)$.

Conclusions-Consistent with the adult literature, pediatric studies demonstrate that treatment with MET results in increased odds of spontaneous ureteral stone passage and a low rate of adverse events. Although the accumulated literature is limited by inconsistent and/or incomplete reporting, there is nonetheless a clear, cumulative positive effect of MET on stone passage among children. The available evidence thus supports a prominent role for MET in treatment algorithms for pediatric urolithiasis.

\section{Keywords}

Pediatric; Kidney stone; Urolithiasis; Nephrolithiasis; Review, Systematic; Meta-Analysis

\section{Introduction}

Kidney stone disease is becoming increasingly common among children and young adults $[1,2]$. Children who develop kidney stones are likely to have recurrent stones, and as such they are at high risk for undergoing multiple stone removal procedures over the course of their lives. Published data would seem to indicate that most children with urolithiasis are treated conservatively with observation to allow spontaneous stone passage, although there is significant inter-institutional variation in surgical treatment rates [3].

In adults, medical expulsive therapy (MET) has been shown to be successful at increasing spontaneous stone passage rates and at reducing the number of stone-related surgical procedures [4,5]. MET typically involves taking a daily medication, usually an alphaadrenergic antagonist such as doxazosin or tamsulosin, to dilate the distal ureter and promote the spontaneous passage of the stone. In a large meta-analysis in adults, MET was found to increase the chances of spontaneous stone passage by $65 \%$ (pooled risk ratio 1.65 , 95\% CI 1.45-1.88) [4]. Whether MET is similarly effective for children, given their relatively smaller ureters, is less clear; few studies have assessed the efficacy of MET in the treatment of urolithiasis in children. The results of these studies are inconsistent and limited by the variation in study designs, patient selection, and outcome measures. Conflicting results have even been reported among the published randomized controlled trials (RCTs) of MET [6-8].

In light of these differences, the goal of this systematic review is to evaluate the accumulated literature on the medical management of pediatric urolithiasis.

\section{Patients and methods}

\section{Search strategy}

We searched the Cochrane Controlled Trials Register, clinicaltrials.gov, MEDLINE, and EMBASE electronic databases for studies published between January 1990 and October 2013 in any language based upon PRISMA guidelines [9]. This date range was chosen to 
provide a contemporary selection of studies. We used the exploded search terms: "urolithiasis", "nephrolithiasis", "kidney stone", or "stone". These were then restricted to articles retrieved under a second search for the exploded search terms "pediatric", "child", or "children".

Reference lists of included studies were manually screened for any additional studies. We also manually searched for unpublished abstracts presented at relevant scientific meetings: American Urological Association, Society for Pediatric Urology, American Academy of Pediatrics Section on Urology, Pediatric Academic Societies, World Congress of Endourology, Société Internationale d'Urologie, and the European Association of Urology. The protocol was prospectively registered at PROSPERO (CRD42013005960).

\section{Selection criteria}

Inclusion criteria were children (aged $\leq 18$ years) with urolithiasis treated with medications with the specific goal of increasing spontaneous stone passage rate, including but not limited to alpha-adrenergic blockers (e.g., tamsulosin or doxazosin), calcium channel blockers (e.g., nifedipine), or other adjuvant medications (e.g., steroids or tolterodine). These patients were then compared against children with urolithiasis undergoing no treatment or other non-MET drug therapy (e.g., non-steroidal anti-inflammatory drug [NSAID]).

Our goal was to include only RCTs; however, we a priori decided that if few pediatric RCTs met inclusion criteria, we would also include observational studies, provided that data from a comparison group was reported. RCT and cohort studies were analyzed as subsets and reported separately. Inclusion criteria included report of the number of patients treated and the fraction for which the treatment was successful. No manuscript was excluded based on method of analysis, definition of success, language of publication, or perceived quality/ susceptibility to bias. In cases of ambiguity or where study reporting made evaluation difficult, we attempted to err on the side of inclusiveness.

\section{Data abstraction}

Two reviewers (N.V. and D.Z.) independently reviewed all study abstracts in duplicate with disagreements resolved by the senior author (J.C.R.). Full text articles appearing to meet selection criteria were reviewed, and study data was abstracted in the same manner. Manuscripts published in languages other than English were translated by study authors fluent in that language and/or by institutional translation staff. Abstracted data included patient-level factors (patient age, stone size, stone passage rate, time to passage, MET agent, adverse events) and study-level factors (study design, country of origin, conflict of interest disclosure, funding). COI was identified by disclosure publication. Our primary outcome was spontaneous stone passage.

\section{Risk of bias assessment}

Bias assessment was undertaken using the Cochrane Collaboration checklist. Funnel plots were visually assessed for evidence of publication bias. Bias assessment did not influence the planned meta-analysis. 


\section{Statistical methods}

Descriptive statistical analyses were performed as appropriate. For univariate pooling, standard Dersimonian-Laird random-effects models were constructed [10]. Study heterogeneity was assessed using the Higgins-Thompson method [11]. Given the small number of eligible studies, meta-regression was not performed.

Influence analyses were performed by sequentially removing individual studies and thus verifying that the effect estimates had not significantly changed. No significant differences with inclusion/exclusion of any study were noted.

All statistical analyses were performed using STATA/SE version 11.0 (College Station, TX, USA) and RevMan version 5.3.5 (Nordic Cochrane Centre of the Cochrane Collaboration, Copenhagen, Denmark).

\section{Results}

\section{Search results}

A total of 11,197 studies were identified using our search criteria. Of these, 37 studies were selected for full review; five studies met all criteria and were included in the pooled qualitative review and the quantitative meta-analysis (Fig. 1). RCTs and retrospective cohort studies were analyzed separately (Figs 2 and 3 ).

\section{Description of studies}

Of the five included studies, three were RCTs [6-8] and two were retrospective cohort studies $[12,13]$. These studies are reported separately below. Of the retrospective cohorts, one has thus far only been reported as an abstract [12] and the other included data on both an unmatched control group (174 children) and a propensity score-matched control group (99 children) [13]. We elected to include the total cohort instead of the matched group.

A total of 465 patients were included in the final systematic review (Table 1). The RCTs included significantly fewer patients than did the retrospective cohort studies (mean 48 vs. $160, p=0.03$ ). Mean patient ages ranged from 5.6 to 14.5 years. Included study populations were from Turkey, Egypt, and the United States. No study reported a significant COI, although COI data was not reported on the unpublished abstract [12]. Although we did not restrict the type of MET that could be offered in order to meet inclusion criteria, all of the studies exclusively used alpha-adrenergic blockers, either tamsulosin or doxazosin. The planned duration of MET prior to surgical intervention ranged from 3-6 weeks for all studies.

\section{Effects of interventions}

Alpha-adrenergic blockers were shown to significantly increase the odds of spontaneous passage of ureteral stones $(<10 \mathrm{~mm})$ in children. This effect appeared more pronounced among the RCTs than among the retrospective cohort studies, although the effect was independently significant for both study designs (OR 4.06, 95\% CI 1.84-8.95, $p=0.0005$ ) vs. $1.83,95 \%$ CI $1.20-2.79, p=0.005)$. 
The between-study heterogeneity was not significant among all studies $\left(I^{2}=14 \%, p=0.36\right)$. Predictably, there was increased heterogeneity noted between the RCT and retrospective studies, although with the small number of studies this did not reach statistical significance $\left(I^{2}=72 \%, p=0.06\right)$.

\section{Risk of bias in included studies}

Concerns about biases affecting the published outcomes of the included studies exist due to the low quality of the RCTs reviewed for analysis (Fig. 4). There was little visual evidence of publication bias noted on the funnel plot (not shown), as confirmed by Begg's test ( $p=$ 0.5 ). Selection bias could not be ruled out in any studies; for example, descriptions of the randomization procedure were not included in two of the RCTs and the cohorts in the retrospective studies are at high risk of selection bias by nature of the study design. Concealment of allocation could not be verified in any of the included studies. Additionally, blinding of study participants, study personnel, or outcome assessors was not reported, potentially leading to problems with performance and detection biases. This particularly puts into question outcomes that have subjective assessments such as the claim by some studies that alpha-adrenergic blocker therapy led to a reduced number of pain episodes during treatment $[7,8]$. Attrition bias was not judged to be of great concern as follow-up of included patients was consistently performed and very few patients withdrew from either the control or treatment groups [10]. This is likely in direct relation to the fact that treatment with alphaadrenergic blockers has such a low side effect profile and that MET exposure was capped in most studies at 3 weeks.

\section{Adverse effects of MET}

Side effects of MET were reported to be minimal. Four out of the five studies reported withdrawal or adverse outcome data; only one out of 175 patients $(0.6 \%)$ who received alpha-adrenergic blocker treatment withdrew from treatment due to adverse effects.

Relatively few patients reported any adverse effects at all; the most commonly reported issue was somnolence, reported in one study to be experienced by $24.2 \%$ of the treatment group versus $17.9 \%$ of the control group [8]. By contrast, the largest included study reported that no patients treated with tamsulosin reported any adverse effects [13].

\section{Discussion}

Consistent with the adult literature, pooled results of pediatric studies demonstrate that MET results in increased ureteral stone passage (OR 4.1 for RCTs). We also found MET to have a low rate of adverse events among all included studies. Reported events were mild, with only one child withdrawing from study due to medication side effects (somnolence, nausea, and vomiting). Given that MET has a relatively high success rate, a low rate of adverse events, and a substantially lower cost than surgical treatments, these results suggest that in children without contraindications a trial of MET should be considered as a first line treatment.

To date, the accumulated literature has been inconsistent in its support of MET for children. In a retrospective case series (which to date has been published only in its abstract form), George et al. [12] found no difference between children taking tamsulosin to those on no 
therapy. The average stone size was $<5 \mathrm{~mm}$ in this study, which in adults has been shown to have a high rate of spontaneous stone passage regardless of therapy [14,15]. This was consistent with a small RCT reported by Aydogdu et al. [6], in which no difference in stone expulsion rate was noted between children taking doxazosin compared to children receiving pain management with ibuprofen.

By contrast, Mokhless reported that tamsulosin increased the spontaneous expulsion rate (87.8\% in the treatment group vs. $64.2 \%$ in the control group) for ureteral stones [8]. This study focused on distal ureteral stones, but the results are limited by the fact that half of the treatment group had received prior treatment with shockwave lithotripsy or percutaneous lithotripsy prior to randomization. Thus, these patients were clearing residual stones, which may have different characteristics than intact, primary stones. More recently conducted studies have also demonstrated an increase in spontaneous stone passage in children treated with alpha-adrenergic blockers [7,13].

Only two studies included stones in the mid or proximal ureter; thus most of the included data in this report reflects stone passage rates for distal ureteral stones. In most included studies, both control and treatment groups received NSAID therapy for symptomatic relief of renal colic. The question of whether NSAID therapy could increase the rate of stone expulsion was previously addressed by at least one RCT that showed that there was no difference in stone passage rates between NSAID and control patients [16].

We noted significant concerns about biases affecting the published outcomes of the included studies (Fig. 4). Selection bias could not be ruled out in most studies as descriptions of the randomization procedure were not included in any of the RCTs, and the cohorts in the retrospective studies were noted to be at high risk of selection bias by nature of the study design. Concealment of allocation could not be verified in any of the included studies. Additionally, blinding of study participants, study personnel, or outcome assessors was not reported, potentially leading to problems with performance and detection biases. This particularly puts into question outcomes that have subjective assessments, such as the claim by some studies that alpha-adrenergic blocker therapy led to a reduced number of pain episodes during treatment $[7,8]$. Attrition bias was not judged to be of great concern as follow-up of included patients was consistently performed and very few patients withdrew from either the control or treatment groups [10]. This is likely in direct relation to the fact that treatment with alpha-adrenergic blockers has such a low side effect profile and that follow-up times were capped in most studies to 3 weeks. Reporting biases could play an important role in our pooled assessment; for example, it is possible that less effective MET is not reported in the literature.

Between-study effect heterogeneity was very low in our pooled analysis despite the fact that the studies came from several different countries and that different medications were used. A possible explanation is that the Higgins-Thompson $I^{2}$ statistic is underpowered in our meta-analysis due to the low number of included studies. However, it is worth noting that heterogeneity was, as expected, markedly higher between the RCT and retrospective study designs $\left(I^{2}=72 \%\right)$. Moreover, the treatment effect was substantially greater in the RCTs than in the retrospective cohort studies (Figs 2 and 3), as would be expected based on the 
characteristics of the study designs. In general, RCTs tend to demonstrate the efficacy of treatments under ideal circumstances, while cohort studies tend to demonstrate the effectiveness of treatments under real-world conditions, such as poor compliance with study medications, or non-ideal patient selection.

As with any systematic review, our analyses were limited by the available data from the included studies. As seen in Fig. 4, published studies of MET were judged to universally be at high risk or, at best, at uncertain risk of significant bias. Studies that report their findings in a straightforward manner such that their data are easily amenable to systematic review are rare. As with any meta-analysis, we must therefore rely on a potentially imperfect data set. Nevertheless, to the best of our knowledge, these are the best data currently available, and these data do suggest a role for MET in the management of children with urolithiasis.

\section{Conclusions}

Consistent with the adult literature, pediatric studies demonstrate that treatment with MET results in increased odds of spontaneous ureteral stone passage and a low rate of adverse events. Although the accumulated literature is limited by inconsistent and/or incomplete reporting, there is nonetheless a clear, cumulative positive effect of MET on stone passage among children. The available evidence thus supports a prominent role for MET in treatment algorithms for pediatric urolithiasis.

\section{Acknowledgments}

Funding: Dr. Routh is supported by grants K12-DK100024 \& K08-DK100534 from the National Institute of Diabetes and Digestive and Kidney Diseases. The funding sources had no role in the collection, analysis and interpretation of data; in the writing of the manuscript; or in the decision to submit the manuscript for publication.

Dr. Wiener has served as a consultant for Eli Lilly \& Company.

\section{References}

1. Dwyer ME, Krambeck AE, Bergstralh EJ, Milliner DS, Lieske JC, Rule AD. Temporal trends in incidence of kidney stones among children: a 25-year population based study. J Urol. 2012; 188:247-252. [PubMed: 22595060]

2. Routh JC, Graham DA, Nelson CP. Epidemiological trends in pediatric urolithiasis at United States freestanding pediatric hospitals. J Urol. 2010; 184:1100-1104. [PubMed: 20650479]

3. Routh JC, Graham DA, Nelson CP. Trends in imaging and surgical management of pediatric urolithiasis at American pediatric hospitals. J Urol. 2010; 184:1816-1822. [PubMed: 20728146]

4. Hollingsworth JM, Rogers MA, Kaufman SR, Bradford TJ, Saint S, Wei JT, et al. Medical therapy to facilitate urinary stone passage: a meta-analysis. Lancet. 2006; 368:1171-1179. [PubMed: 17011944]

5. Singh A, Alter HJ, Littlepage A. A systematic review of medical therapy to facilitate passage of ureteral calculi. Ann Emerg Med. 2007; 50:552-563. [PubMed: 17681643]

6. Aydogdu O, Burgu B, Gucuk A, Suer E, Soygur T. Effectiveness of doxazosin in treatment of distal ureteral stones in children. J Urol. 2009; 182:2880-2884. [PubMed: 19846149]

7. Erturhan S, Bayrak O, Sarica K, Seckiner I, Baturu M, Sen H. Efficacy of medical expulsive treatment with doxazosin in pediatric patients. Urology. 2013; 81:640-643. [PubMed: 23343614]

8. Mokhless I, Zahran AR, Youssif M, Fahmy A. Tamsulosin for the management of distal ureteral stones in children: a prospective randomized study. J Pediatr Urol. 2012; 8:544-548. [PubMed: 22099477] 
9. Moher D, Liberati A, Tetzlaff J, Altman DG, Group P. Preferred reporting items for systematic reviews and meta-analyses: the PRISMA statement. Ann Intern Med. 2009; 151:264-269. W64. [PubMed: 19622511]

10. DerSimonian R, Laird N. Meta-analysis in clinical trials. Control Clin Trials. 1986; 7:177-188. [PubMed: 3802833]

11. Higgins JP, Thompson SG, Deeks JJ, Altman DG. Measuring inconsistency in meta-analyses. BMJ. 2003; 327:557-560. [PubMed: 12958120]

12. George, A.; Montag, S.; Cubillos, J.; Gitlin, J.; Palmer, LS. The Effect of Tamsulosin on Ureterolithiasis in the Pediatric Population; American Urological Association Annual Meeting; Washington, DC. 2011.

13. Tasian GE, Cost NG, Granberg CF, Pulido JE, Rivera M, Schwen Z, et al. Tamsulosin and the spontaneous passage of ureteral stones in children: A multi-institutional cohort study. J Urol. 2014; 192:506-511. [PubMed: 24518765]

14. Preminger GM, Tiselius HG, Assimos DG, Alken P, Buck C, Gallucci M, et al. 2007 guideline for the management of ureteral calculi. J Urol. 2007; 178:2418-2434. [PubMed: 17993340]

15. Segura JW, Preminger GM, Assimos DG, Dretler SP, Kahn RI, Lingeman JE, et al. Ureteral Stones Clinical Guidelines Panel summary report on the management of ureteral calculi. The American Urological Association. J Urol. 1997; 158:1915-1921. [PubMed: 9334635]

16. Laerum E, Ommundsen OE, Gronseth JE, Christiansen A, Fagertun HE. Oral diclofenac in the prophylactic treatment of recurrent renal colic. A double-blind comparison with placebo. Eur Urol. 1995; 28:108-111. [PubMed: 8529732] 


\section{HIGHLIGHTS}

- We identified 11,197 studies, 5 of which (3 randomized controlled trials, 2 retrospective cohorts) were included in the pooled meta-analysis.

- We found little evidence of significant publication bias, but we were unable to assess the likelihood of other forms of bias (allocation, selection) for most included studies due to reporting limitations.

- Pooled results demonstrate that medical expulsive therapy significantly increased the odds of spontaneous stone passage (OR 2.21, 95\% CI 1.40-3.49).

- Relatively few patients reported any adverse effects at all; the most commonly reported issue was somnolence. 


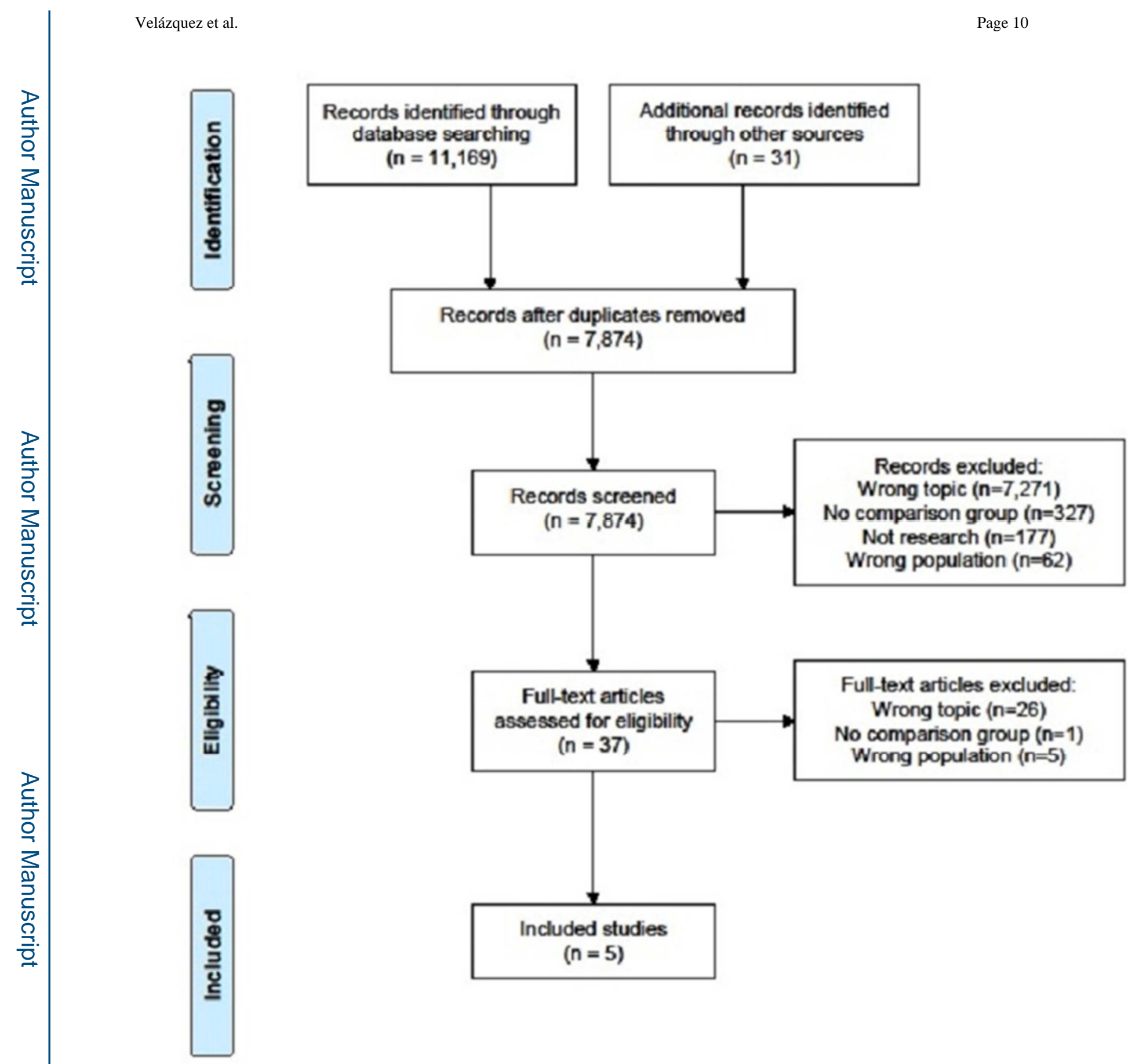

Figure 1.

PRISMA Flow Diagram for the Systematic Review 


\begin{tabular}{|c|c|c|c|c|c|c|}
\hline \multirow[b]{2}{*}{ Study or Subgroup } & \multicolumn{2}{|c|}{ MET } & \multicolumn{2}{|c|}{ Control } & \multirow[b]{2}{*}{ Weight } & \multirow{2}{*}{$\begin{array}{c}\text { Odds Ratio } \\
\text { M-H, Random, 95\% C }\end{array}$} \\
\hline & Events & Total & Events & Total & & \\
\hline Aydogdu 2009 & 16 & 19 & 14 & 20 & $25.7 \%$ & $2.29[0.48,10.88]$ \\
\hline Ertuhan 2013 & 17 & 24 & 6 & 21 & $37.4 \%$ & $6.07[1.67,22.12]$ \\
\hline Mokhless 2012 & 29 & 33 & 18 & 28 & $37.0 \%$ & $4.03[1.10,14.78$ \\
\hline Total $(95 \% \mathrm{CI})$ & & 76 & & 69 & $100.0 \%$ & $4.06[1.84,8$. \\
\hline $\begin{array}{l}\text { Total events } \\
\text { Heterogeneity. Tau }{ }^{2} \\
\text { Test for overall effec }\end{array}$ & $\begin{aligned} & 62 \\
& 00 ; C h \\
&=3.47\end{aligned}$ & $P=$ & $\begin{array}{r}38 \\
89, \mathrm{df}= \\
.0005)\end{array}$ & $17=$ & 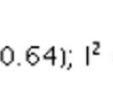 & $\%$ \\
\hline
\end{tabular}

Figure 2.

Forest Plot of RCT Studies 


\begin{tabular}{|c|c|c|c|c|c|c|}
\hline \multirow[b]{2}{*}{ Study or Subgroup } & \multicolumn{2}{|c|}{ MET } & \multicolumn{2}{|c|}{ Control } & \multirow[b]{2}{*}{ Weight } & \multirow{2}{*}{$\begin{array}{c}\text { Odds Ratio } \\
\mathrm{M}-\mathrm{H} \text {, Random, } 95 \% \mathrm{CI}\end{array}$} \\
\hline & Events & Total & Events & Total & & \\
\hline George 2011 & 25 & 37 & 44 & 85 & $27.5 \%$ & $1.94[0.86,4.36]$ \\
\hline Tasian 2014 & 55 & 99 & 72 & 175 & $72.5 \%$ & $1.79[1.09,2.94]$ \\
\hline Total $(95 \% \mathrm{CI})$ & & 136 & & 260 & $100.0 \%$ & $1.83[1.20,2.79]$ \\
\hline $\begin{array}{l}\text { Total events } \\
\text { Heterogeneity. Tau } \\
\text { Test for overall effec }\end{array}$ & $\begin{aligned} & 80 \\
& .00 ; \mathrm{Ch} \\
&= 2.79\end{aligned}$ & 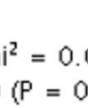 & $\begin{array}{l}116 \\
\text { 3f }= \\
051\end{array}$ & 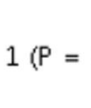 & 0.87 & $0 \%$ \\
\hline
\end{tabular}

Figure 3.

Forest Plot of Retrospective Cohort Studies 


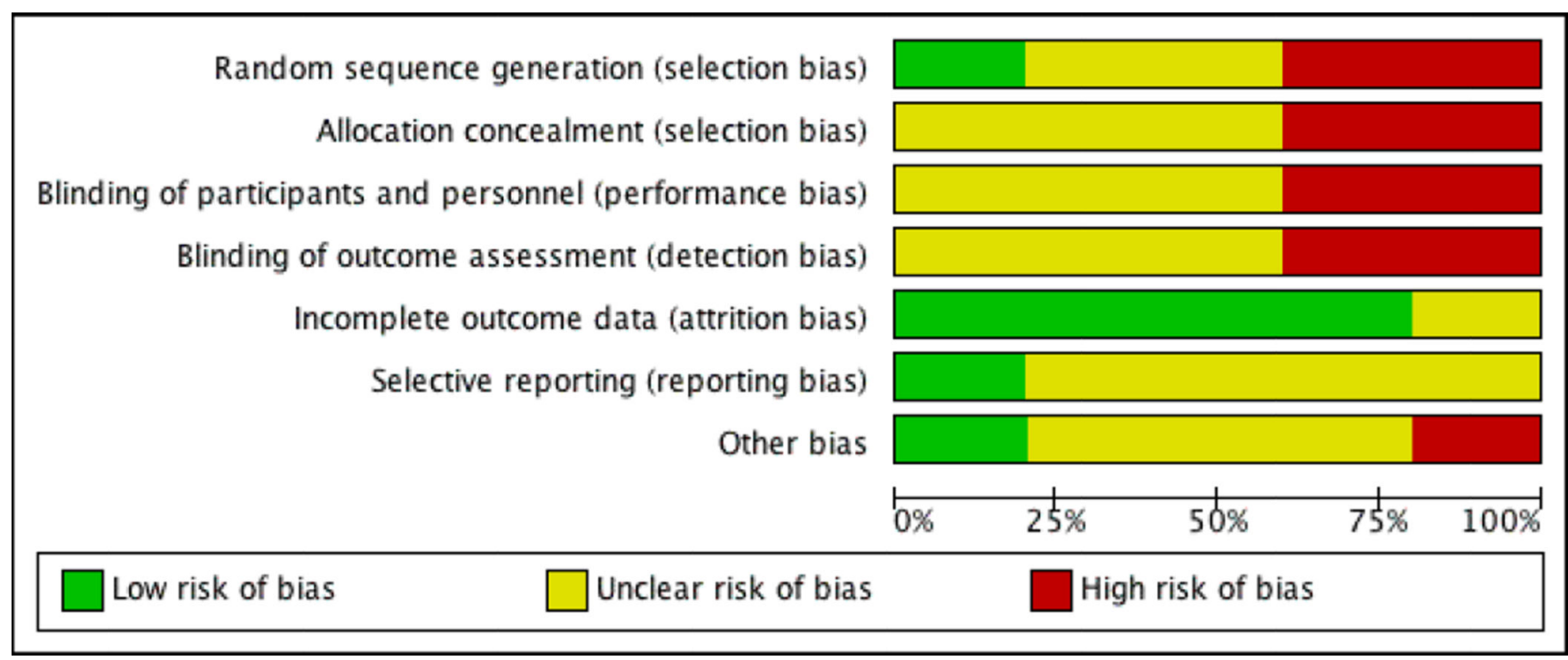

Figure 4.

Cochrane Risk of Bias Graph; this graph shows the fraction of all studies that are at high, intermediate, and low risk of bias in various areas. 


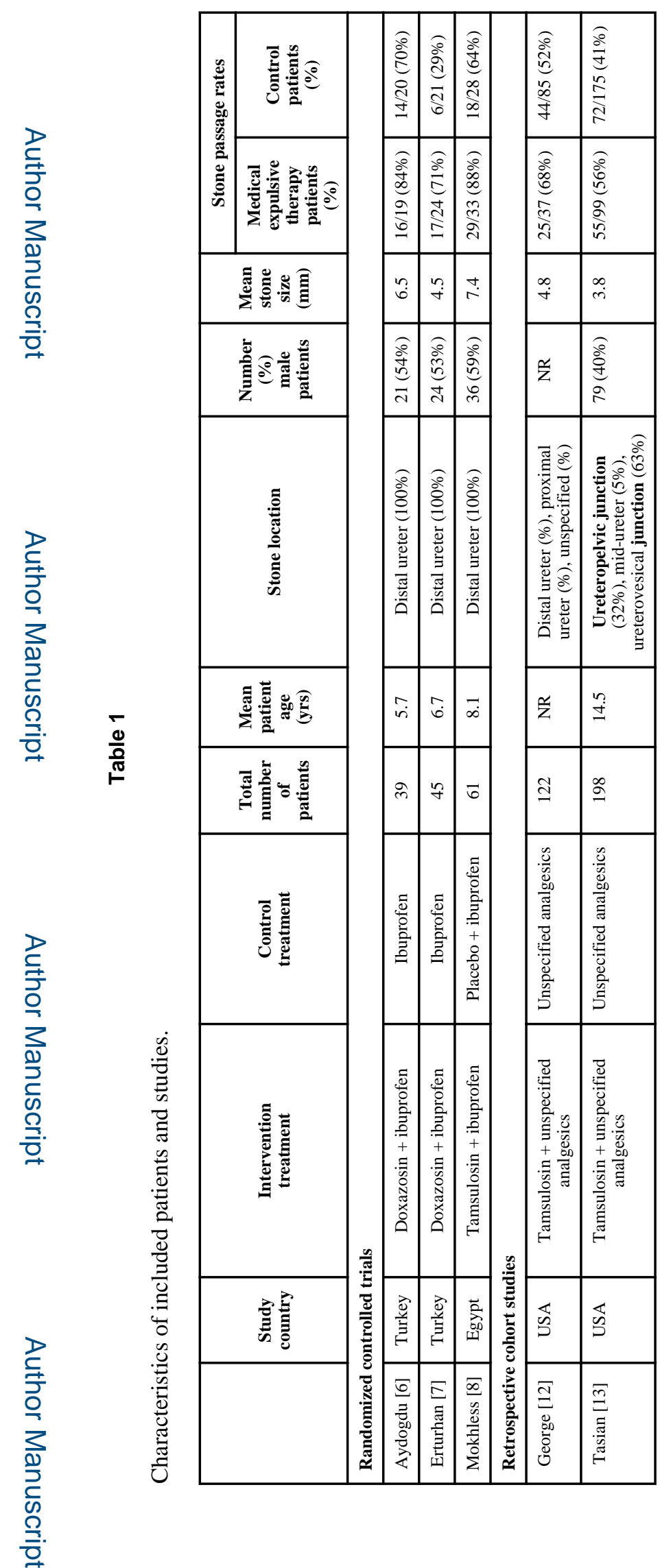

J Pediatr Urol. Author manuscript; available in PMC 2016 December 01. 